\title{
Multi-parameter uncertainty analysis of a bifurcation point
}

\author{
B. Knopf ${ }^{1}$, M. Flechsig ${ }^{1}$, and K. Zickfeld ${ }^{2}$ \\ ${ }^{1}$ Potsdam Institute for Climate Impact Research, P.O. Box 6012 03, 14412 Potsdam, Germany \\ ${ }^{2}$ School of Earth and Ocean Sciences, University of Victoria, Victoria, BC, Canada
}

Received: 29 June 2006 - Revised: 6 October 2006 - Accepted: 6 October 2006 - Published: 10 October 2006

\begin{abstract}
Parameter uncertainty analysis of climate models has become a standard approach for model validation and testing their sensitivity. Here we present a novel approach that allows one to estimate the robustness of a bifurcation point in a multi-parameter space. In this study we investigate a box model of the Indian summer monsoon that exhibits a saddle-node bifurcation against those parameters that govern the heat balance of the system. The bifurcation brings about a change from a wet summer monsoon regime to a regime that is characterised by low precipitation. To analyse the robustness of the bifurcation point itself and its location in parameter space, we perform a multi-parameter uncertainty analysis by applying qualitative, Monte Carlo and deterministic methods that are provided by a multi-run simulation environment.

Our results show that the occurrence of the bifurcation point is robust over a wide range of parameter values. The position of the bifurcation, however, is found to be sensitive on these specific parameter choices.
\end{abstract}

\section{Introduction}

The Indian summer monsoon (ISM) is a yearly recurring phenomenon that brings vital rain to India. The ISM develops in summer due to thermal gradients between the warm Indian continent and the cooler temperatures over the Indian Ocean. The warmer air masses over land result in areas of low pressure whereas over the ocean the colder air masses yield higher pressures. This pressure gradient entails the supply of moisture over the Indian continent that ascends, condensates, and is released as precipitation.

The influence of anthropogenic activities on the ISM has been investigated in a number of studies. Most of them indicate that emissions of greenhouse gases, that alter the heat

Correspondence to: B. Knopf

(brigitte.knopf@pik-potsdam.de) budget of the system and therefore the land-sea temperature contrast, could increase the ISM intensity and/or variability (Meehl and Washington, 1993; Zwiers and Kharin, 1998; May, 2002, e.g.). On the other hand, there is evidence of aerosol-induced reduction of precipitation as well (Patra et al., 2005, e.g.) or of no clear indication for an influence of global warming at all (Kripalani et al., 2003). In a study of a model of the ISM it was shown that the monsoon can undergo a transition from a wet to a dry regime due to anthropogenic activity (Zickfeld et al., 2005), which would have dramatic effects for the population of India. The most important finding in this model is a saddle-node bifurcation against the planetary albedo, that is the albedo at the top of the atmosphere, and other parameters that influence the heat budget of the system. As the planetary albedo may be modified by human activities, such as emissions of scattering aerosols and land-cover conversion, there is concern that this regime shift could be triggered under global change. Palaeoclimate data indicate that such a regime transition may have occurred during the last glacial period (Burns et al., 2003) and the Holocene (Gupta et al., 2003). To test the robustness of the bifurcation it is indispensable to accomplish a multiparameter uncertainty analysis to investigate the dependence of the model results from the choice of the parameters. Moreover it is desirable to explore the influence of anthropogenic perturbations on the position of the bifurcation point.

In the recent years multi-parameter uncertainty analysis has increasingly become a standard approach in climate modelling to quantify model uncertainty. By a multi-parameter uncertainty analysis we mean an analysis where several parameters are varied simultaneously to cover the whole range of possible parameter combinations. As this is a very time consuming and computationally expensive procedure in large models, only a few parameters are normally varied. A detailed parameter study with 11 parameters has been performed by Schneider von Deimling et al. (2006) with the CLIMBER-2 model of intermediate complexity to reduce

Published by Copernicus GmbH on behalf of the European Geosciences Union and the American Geophysical Union. 


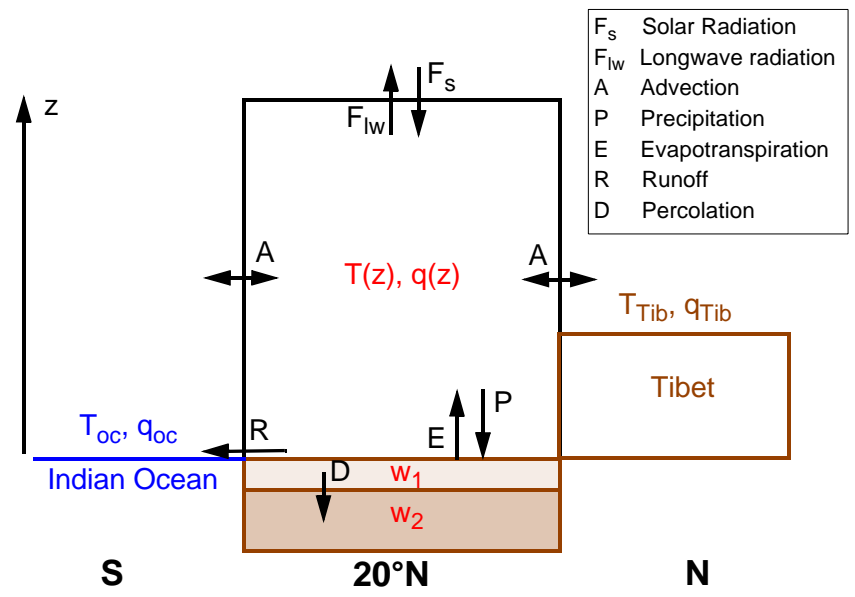

Fig. 1. Schematic view of the box model of the Indian monsoon. $T$ denotes temperature, $q$ specific humidity. $w_{1}$ and $w_{2}$ are the soil moistures in the upper and lower soil layer. The variables inside the box $\left(T(z), q(z), w_{1}, w_{2}\right)$ are calculated prognostically, whereas the climatic conditions at the box boundaries $\left(T_{i}, q_{i}\right.$, with $i \in\{o c, T i b\})$ are externally prescribed.

the uncertainty range for climate sensitivity, defined as the change in equilibrium global mean temperature following a doubling of atmospheric $\mathrm{CO}_{2}$ content. Similar studies were carried out by Knutti et al. (2002) and Forest et al. (2002). Stainforth et al. (2005) studied the sensitivity of a general circulation model (GCM) by varying six parameters. In our case the challenge is to expand such an analysis by providing not only the multi-parameter setting but additionally performing a bifurcation analysis. On the other hand, we have the advantage that our model is a conceptual one that requires only limited computational resources. The innovative point is that we investigate the bifurcation point in the whole parameter space and not just in dependence on two parameters, as it is mainly done in other bifurcation uncertainty studies, e.g. by Kumar Mittal et al. (2005) for a paradigmatic monsoon model or Longworth et al. (2005) for a box model of the thermohaline circulation. Another approach that deals with model uncertainty in relation to bifurcations was presented by Gross and Feudel (2006) and classifies the systems in an abstract way according to their structure. This method, however, is mainly applicable to structural uncertainty whereas here the focus is directly on the parameter uncertainty. The widely used bifurcation analysis program AUTO (Doedel, 1981), that is based on a numerical continuation method, provides the possibility to follow a bifurcation point in two parameter directions. CONTENT (Kuznetsov et al., 1997), another bifurcation analysis software, is indeed able to handle a continuation in three parameter directions, what is still not enough for our model with nearly 60 parameters. To realise the multi-parameter analysis we apply a new method based on the combination of the multi-run simulation environment SimEnv (Flechsig et al., 2005; SimEnv, 2006) with AUTO that will be described in the following. As this method is model-independent and therefore demonstrates a general approach, it can be applied to other models of similar complexity, where the robustness of a certain bifurcation point is of interest.

The paper is organised as follows: in Sect. 2 we introduce the model and the method for the multi-parameter bifurcation analysis. We will carry out the systematic approach starting with a global sensitivity analysis (cf. Sect. 3.1) to deduce the most influential parameters. We proceed with Monte Carlo analyses that allow to study the error propagation of the parameter uncertainties in the model (cf. Sect. 3.2) that is followed by a deterministic parameter screening (cf. Sect. 3.3) to investigate how the model performs under changes of these parameters. We will focus on the anthropogenically influenced parameters to investigate whether the abrupt regime transition could be relevant under global change and conclude our analysis in Sect. 4.

\section{Model and method}

The model investigated here is a box model of the Indian summer monsoon (Zickfeld et al., 2005; Zickfeld, 2004). The model consists of a three-layer tropical atmosphere over a two-layer soil module. It includes the representations of the hydrological cycle as well as surface hydrology. The prognostic variables in the model are the near-surface air temperature $T_{a}$ and specific humidity $q_{a}$ over India, and the soil moisture of the two soil layers $w_{1}, w_{2}$ (cf. Fig. 1). Variables such as precipitation, evaporation and the monsoon circulation are diagnosed from the above-mentioned four. The climate conditions at the box boundaries for the temperature and the specific humidity are externally prescribed and are fixed to summer conditions.

The temporal evolution of the surface temperature $T_{a}$ and the specific humidity $q_{a}$ are computed from the vertically integrated combined surface-plus-atmospheric heat and the atmospheric water vapour balance equations:

$$
\begin{aligned}
& \int_{0}^{H_{a}} c_{p} \rho \frac{\partial \theta}{\partial t} d z+h_{s} c_{s} \frac{\partial T_{a}}{\partial t}= F_{\downarrow}\left(1-A_{s y s}\right)-F_{\uparrow}+ \\
& A_{T}+\mathcal{L}(C-E), \\
& \int_{0}^{H_{a}} \rho \frac{\partial q}{\partial t} d z=E-C+A_{v},
\end{aligned}
$$

where $\theta=T(z)+\Gamma_{a} z$ denotes the potential temperature and $\Gamma_{a}$ the adiabatic lapse rate in the atmosphere. The vertical profile of the temperature $T(z)$ is parameterised in terms of the near surface value $T_{a}$ with a linear profile in $z$ in the troposphere $\left(T(z)=T_{a}-\Gamma z\right)$ and a constant profile in the stratosphere $\left(T(z)=T_{a}-\Gamma H_{t}\right)$ with the lapse rate $\Gamma$, that is modelled as a function of $T_{a}$ and $q_{a}$, and the height of the troposphere $H_{t}$. The specific humidity $q$ is assumed to follow an exponential vertical profile with $q(z)=q_{a} \exp \left(-z / H_{e}\right)$, where $H_{e}$ is the water vapour scale height. $\mathcal{L}$ refers to the 
latent heat of evaporation. The diagnostic variables on the right hand side of the equations denote the following fluxes and sources/sinks and are given with their dependence on the variables:

- $F_{\downarrow}$ : Incident solar radiation at the top of the atmosphere,

- $F_{\uparrow}\left(T_{a}, q_{a}\right)$ : Outgoing radiation at the top of the atmosphere,

- $A_{\text {sys }}\left(T_{a}, q_{a}\right):$ Planetary albedo,

- $E\left(q_{a}, w_{1}\right):$ Evapotranspiration,

- $C\left(T_{a}, q_{a}\right):$ Condensation rate,

- $A_{T}\left(T_{a}, q_{a}\right):$ Horizontal advection of heat,

- $A_{T}\left(T_{a}, q_{a}\right)$ : Horizontal advection of moisture.

Further, $H_{a}$ denotes the height of the atmosphere and $\rho=\rho(z)$ the air density. $c_{p}$ is the specific heat of air, $c_{s}$ the heat capacity per unit soil volume, and $h_{s}$ the depth of the upper soil layer.

The soil moisture is described explicitly according to a two-layer model (Hansen et al., 1986), whereby the water content of the upper layer responds to evapotranspiration $E$ and precipitation $P=P\left(T_{a}, q_{a}\right)$ and the lower layer acts as a reservoir. The rates of change of moisture in the two soil layers are:

$$
\begin{aligned}
\frac{\partial w_{1}}{\partial t} & =\frac{P-E-R}{f_{1}}-\frac{w_{1}-w_{2}}{\tau}, \\
\frac{\partial w_{2}}{\partial t} & =\frac{f_{1}}{f_{2}} \frac{w_{1}-w_{2}}{\tau}
\end{aligned}
$$

where the wetness of the $i$ th layer $w_{i}$ is the ratio of available water to field capacity $f_{i}$. Further, $R=R\left(T_{a}, q_{a}, w_{1}\right)$ is the surface runoff and $\tau$ the time constant for diffusion of moisture between the two layers. Here we introduce in detail only some of the above mentioned diagnostic variables and those parameterisations that are focus of the uncertainty study. A complete description of the model is given in Zickfeld (2004).

The incident solar radiation at the top of the atmosphere $F_{\downarrow}$ is a function of the solar constant and the solar zenit angle. The net outgoing longwave radiation $F_{\uparrow}$ is a function of the atmospheric $\mathrm{CO}_{2}$ concentration, the temperature $T_{a}$ and the cloudiness $N$ :

$$
F_{\uparrow}=A_{0}+B_{0}\left(T_{a}-T_{0}\right)-N\left(C_{0}+D_{0}\left(T_{a}-T_{0}\right)\right),
$$

where $T_{0}$ is the reference temperature and the coefficients $A_{0}, B_{0}, C_{0}, D_{0}$ are functions of the atmospheric $\mathrm{CO}_{2}$ concentration $\mathrm{pCO}_{2}$ in relation to the reference $\mathrm{CO}_{2}$ concentration $p \mathrm{CO}_{2}{ }^{0}$ :

$X_{0}=X_{00}\left(1-\eta \ln \left(\frac{p \mathrm{CO}_{2}}{p \mathrm{CO}_{2}{ }^{0}}\right)\right)$, where $X \in\{A, B, C, D\}$.

The total cloud amount over India $N=N\left(T_{a}, q_{a}\right)$ is a superposition of stratus and cumulus cloud amount, $N_{s t}$ and $N_{c u}$, where the latter is a strongly nonlinear function of the temperature and the specific humidity $T_{a}$ and $q_{a}$ over India. The planetary albedo is the sum of the albedos of stratus clouds $\left(A_{s t}\right)$, cumulus clouds $\left(A_{c u}\right)$ and the clear sky atmosphere $\left(A_{c s}\right)$ :

$A_{s y s}=N_{s t} A_{s t}^{p}+N_{c u} A_{c u}^{p}+(1-N) A_{c s}^{p}$,

where $A_{i}^{p}$ with $i \in\{s t, c u, c s\}$ are functions of the respective albedos $A_{i}$ and the surface albedo $A_{s}$ :

$A_{i}^{p}=\left(A_{i}+\left(1-A_{i}\right)^{2} A_{s}\right) P_{i}$.

$P_{c l}:=P_{s t}=P_{c u}$ and $P_{c s}$ are the integral transmission functions.

The condensation rate $C$ is set equal to the precipitation rate $P$ with

$C=P=\frac{N}{\tau_{P}} \int_{0}^{H_{t}} \rho q d z$,

where $\tau_{P}=\tau_{P}\left(T_{a}\right)$ is the turnover time of water in the atmosphere. The evapotranspiration of the vegetated soil $E$ consists of the contributions from the transpiration from the vegetated soil and the evaporation from the bare soil and depends on the specific humidity $q_{a}$ and the moisture in the upper soil layer $w_{1}$. The runoff $R$ is a function of precipitation $P$ and the soil moisture $w_{1}$.

The horizontal advection terms of heat and moisture, $A_{T}$ and $A_{v}$, are described as the vertically integrated sensible heat and moisture transport due to the horizontal monsoon circulation $u_{m}$, whereby the latter is driven by the temperature contrast between land and ocean $\left(T_{a}-T_{o c}\right)$. In summer, when the land is warmer than the adjacent ocean, i.e. $T_{a}>T_{o c}$, the monsoon circulation $u_{m}$ is directed from the ocean to the Indian peninsula. In winter, i.e. $T_{a}<T_{o c}$, the monsoon cell is reversed.

With this formulation, the surface albedo $A_{s}$ serves as bifurcation parameter, representing a crucial parameter that can be influenced by anthropogenic action. The ISM shows a saddle node bifurcation against $A_{s}$ with a change from a wet to a dry monsoon regime and a certain parameter range where a bistable regime is present (cf. Fig. 2). Similar to the precipitation $P$, the temperature $T_{a}$ decreases with increasing $A_{s}$. Therefore the lower stable branch, emerging at SN2, shows a reversed direction of the monsoon circulation $u_{m}$, i.e. $T_{a}<T_{o c}$, corresponding to winter-like conditions.

For the multi-parameter analysis we first have to identify a (large) set of parameters with their uncertainty ranges. The multi-run simulation environment SimEnv draws samples from this parameter space where each sample corresponds to the goal of a specific sensitivity experiment. SimEnv provides pre-defined experiment types that correspond with methods to deal with model sensitivity and uncertainty, such 


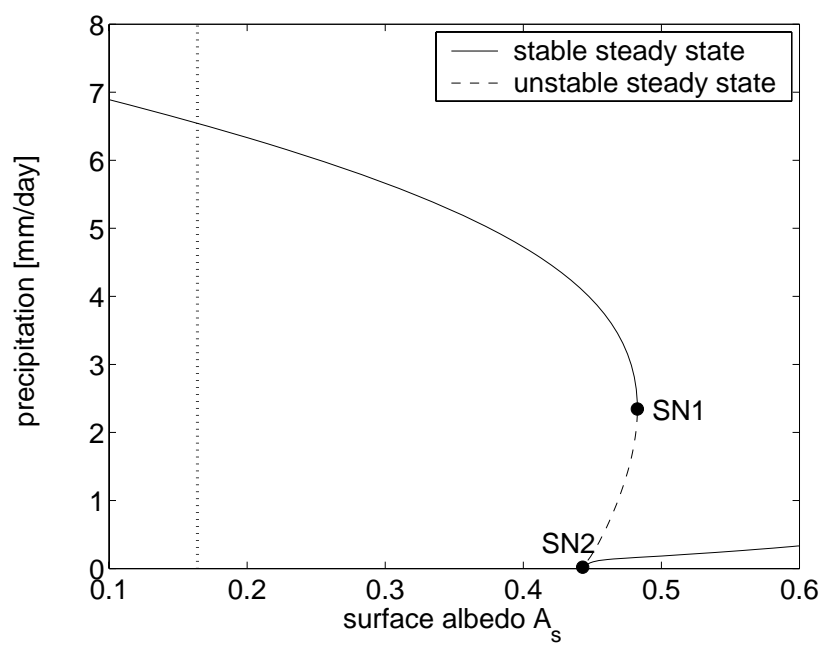

Fig. 2. Bifurcation diagram for the summer monsoon precipitation in dependence of the surface albedo $A_{s}$. The saddle node bifurcation is at $A_{s}=0.483$. The vertical dotted line marks the present day value.

as Monte Carlo techniques or deterministic sensitivity experiments. The model under investigation is interfaced to the simulation environment by minimal source code modifications. As AUTO needs a stable state of the system as a starting point, we first integrate the model until it reaches equilibrium. For each parameter sample point of an experiment we start the qualitative analysis using AUTO with this equilibrium state and run a bifurcation analysis against the specified bifurcation parameter. During interactive experiment postprocessing, the combined state and parameter space is investigated and sensitivity and uncertainty measures are derived. Here sensitivity is related to the bifurcation point SN1 of the system (cf. Fig. 2) and it is analysed whether and at which value - for a certain parameter setting - the saddle node bifurcation occurs. As our model is a conceptual one we have - in contrast to similar studies with GCMs - the advantage that it requires only limited computational resources so we can investigate a large set of parameters and sample points.

We investigate 38 out of 59 model parameters that either influence the heat budget of the system, depend on anthropogenic influence, or are tuneable parameters in the model. The other parameters are physical constants or have only an influence on the transient dynamics but not on the existence of equilibria, as e.g. $f_{2}$ in Eq. (4). We distinguish the parameters that refer to observational quantities (e.g. for the ocean temperature $T_{o c}$ ) from the entire model parameters and determine the uncertainty ranges of the former by analysing the observed values - if they were available - for the period 1948-2004 from the NCAR/NCEP reanalysis (Kalnay et al., 1996) or by including future projections (e.g. for the $\mathrm{CO}_{2}$ concentration $\left.p \mathrm{CO}_{2}\right)$. For the other parameters we simply assume a $\pm 5 \%$ or $\pm 10 \%$ deviation from the default value
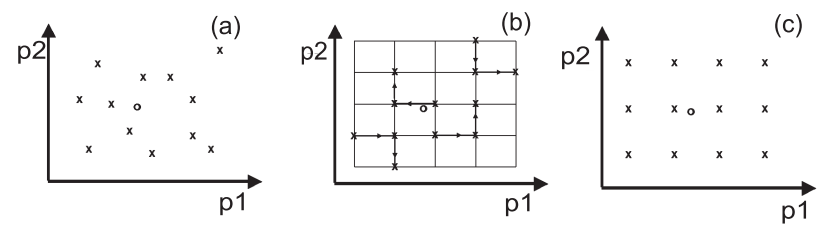

Fig. 3. Applied SimEnv parameter space sampling strategies. Examples for a two-dimensional parameter space $P_{2}=\left(p_{1}, p_{2}\right)$ : (a) Monte-Carlo analysis with a sampling scheme according to probability density functions, (b) global sensitivity analysis to deduce a qualitative ranking of the parameters, (c) deterministic parameter screening to investigate the model's behaviour. The point "o" marks the default value, the crosses " $x$ " denote the sampling points.

in the model. The parameter ranges are chosen such that they are equally likely and associated with the same uncertainty. We assume that the parameters are equally distributed over their ranges, which constitutes of course a certain bias, but as we focus on qualitative changes this assumption does not affect our conclusions. Besides the full parameter set, denoted as $P_{38}$ in the following, we will investigate two 5dimensional subsets. This is the parameter set $P_{S 5}$ including the five most influential parameters and the subset $P_{A 5}$ that denotes five most important anthropogenically influenced parameters.

\section{Multi-parameter uncertainty analysis}

\subsection{Global sensitivity analysis}

The main idea of the global sensitivity analysis (GSA) is to identify those parameters that influence the position of the bifurcation point the most (the least) and to come up with a qualitative ranking of the parameters according to their importance. The method applied by SimEnv is the Morris method (Morris, 1991), modified by Campolongo et al. (2005). For the GSA we investigate the parameter set $P_{38}$ with the specified uncertainty ranges that are subdivided into $m=6$ equal intervals for each parameter. On the resulting regular grid of $38^{m+1}$ grid points the GSA lays randomly trajectories through this 38 -dimensional space, whereby two adjacent points on a trajectory only differ in one parameter $p_{i}$. The points are simultaneously adjacent grid points and each $p_{i}$ is varied only once on every trajectory. In the parameter space this results in trajectories with $38+1$ points. For $m=4$ and two parameters this is exemplarily shown in Fig. $3 \mathrm{~b}$ for four trajectories. Measured is the distance $\Delta F_{i j}$ between the two values for the surface albedo $A_{s}$ at the bifurcation point of the $j^{\text {th }}$ trajectory with respect to the parameter $p_{i}$ that is varied. Two statistical measures are derived for each parameter $p_{i}$ from this analysis: the mean absolute value $\mu_{i}=$ mean $\left(\left|\Delta F_{i}\right|\right)$ and the standard deviation $\sigma_{i}=\sigma\left(\Delta F_{i}\right)$ taken over all $j$ trajectories. A high mean value 


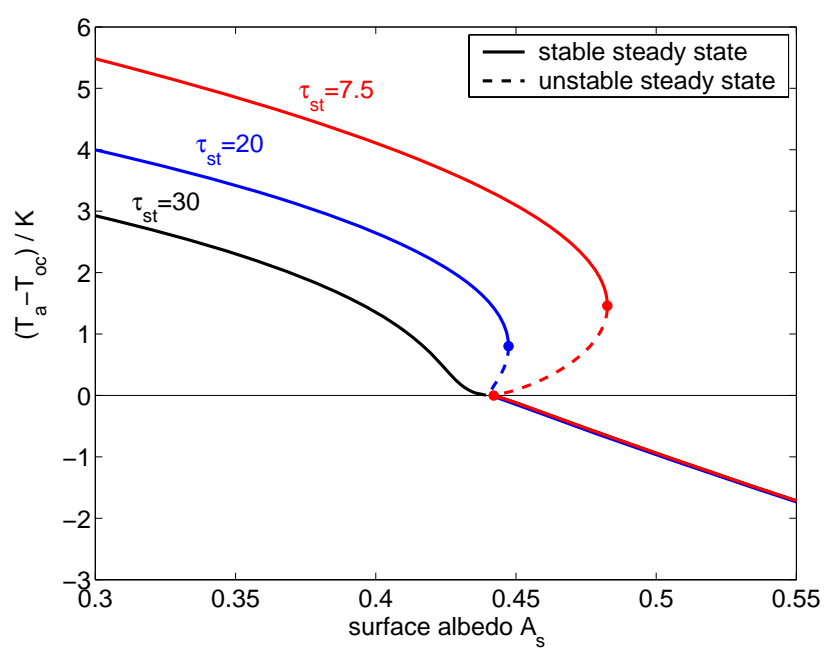

Fig. 4. Comparision of the bifurcation diagram for the temperature difference $\left(T_{a}-T_{o c}\right)$ in dependence on different values of the parameter $\tau_{s t}$. For a value of $\tau_{s t}=30$, the saddle node bifurcation SN1 cannot be detected any more.

$\mu_{i}$ is interpreted as an important overall influence of the parameter on the model output. A high variance $\sigma_{i}$ indicates that the parameter is involved in interactions with other parameters with respect to the model output or that the effect of the parameter on the model output is non-linear (Saltelli et al., 2004). The values for $\mu_{i}$ can directly be interpreted in terms of the bifurcation point: on average the saddle node is shifted about $\mu_{i}$ units when the parameter $p_{i}$ is varied from one grid point to an adjacent one in the given parameter range.

Here we randomly sample 1000 trajectories, resulting in 39000 sample points and single model runs in total, of which 2714 do not show a saddle node bifurcation. In these cases the bifurcation point is not reached before the temperature $T_{a}$ falls below the ocean temperature $T_{o c}$ and the regime shifts from summer to winter-like conditions with a reversal of the temperature difference $\left(T_{a}-T_{o c}\right)$, cf. Fig. 4 . This means that in $93 \%$ of the runs a saddle node bifurcation occurs, leading to the conclusion that the bifurcation point is a very robust feature of the model and not just due to a specific choice of parameters.

Figure 5 displays the ranking according to the value of $\mu$ and $\sigma$ resulting from this analysis. The ranking obviously depends on the choice of the parameter ranges. However, by performing a second GSA with slightly different ranges for some of the parameters, we could confirm the ranking from Fig. 5 with only minor exceptions. As the choice of the uncertainty range is somewhat arbitrary, the absolute value of the mean distance $\mu$ has to be considered in the interpretation as well. This all the more, as we obtain a clustering of the parameter sensitivities with only a few very sensitive parameters and a large cluster for the others (cf. Fig. 5). The

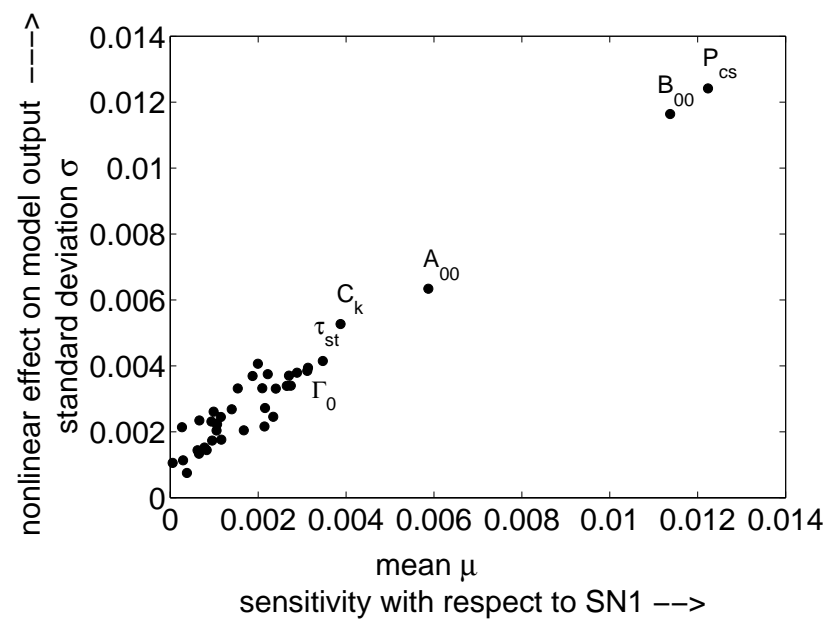

Fig. 5. Results from the GSA with 39000 runs with respect to the value of $A_{s}$ at the bifurcation point. $\mu$ and $\sigma$ are explained in the text. The six most influential parameters are marked.

GSA allows us to deduce two parameter subsets: The set of the five most influential parameters, where we omit here the parameter $A_{00}$ as it describes the same physical process as $B_{00}$ (outgoing longwave radiation, cf. Eqs. (5) and (6)). The subset $P_{A 5}$ that include the five most influential parameters that are subject to anthropogenic disturbances listed in Table 2 .

Categorising the parameters by their influence on certain variables or processes, it attracts attention that those parameters are highly influential, which determine the planetary albedo $A_{s y s}$ through the albedos of the clear sky (influenced by the parameter $P_{c s}$, cf. Eq. (8)) and the clouds $\left(\tau_{s t}\right.$, a parameter determining the albedo of stratus clouds $A_{s t}$, cf. Eq. (8)). $B_{00}$ affects the outgoing longwave radiation at the top of the atmosphere $F_{\uparrow}$ in Eq. (1), cf. Eqs. (5) and (6). $C_{k}$ determines the monsoon circulation and therefore the advection of heat and moisture $A_{T}$ and $A_{v}$, cf. Eqs. (1) and (2), and the coefficient $\Gamma_{0}$ determines the lapse rate $\Gamma$ and therefore the heat budget of the system as well.

\subsection{Monte Carlo analysis}

A Monte Carlo analysis (MCA, cf. Fig. 3a) relates uncertainties in parameter values to uncertainty in an output quantity and therefore demonstrates the error propagation through a model. We implement a Latin hypercube sampling (McKay et al., 1979), to reduce the total randomness. For all three parameter sets $P_{38}, P_{S 5}$, and $P_{A 5}$ we performed 20000 runs where for $P_{38} 96.4 \%$ and for $P_{S 5}$ and $P_{A 5}$ all of the runs showed a saddle node bifurcation. This again confirms that the saddle node bifurcation is a very robust result in this model under parameter uncertainty. For $P_{38}$ the distribution of the bifurcation points shows approximately a normal distribution around the mean value of $A_{s}=0.486$ (cf. 
Table 1. Parameter set $P_{S 5}$ with the ranges that are chosen for the sensitivity analyses. The entries in this list are ranked according to their importance deduced from the GSA: the first parameter is the most sensitive, the last one with the least influence. $\mu$ is the mean of the absolute distance $\left|\Delta F_{i}\right|$ (see text) with respect to the particular parameter.

\begin{tabular}{llll}
\hline \multicolumn{2}{c}{ parameter [unit] } & $\begin{array}{l}\text { range } \\
\text { in [unit] }\end{array}$ & $\begin{array}{l}\mu \\
{\left[10^{-3}\right]}\end{array}$ \\
\hline$P_{c s}$ & integral transmission func. in clear-sky atm. [1] & $0.72-0.86$ & 12.23 \\
$B_{00}$ & parameter of the outgoing radiation $\left[\mathrm{W} / \mathrm{m}^{2} / \mathrm{K}\right]$ & $1.6-2.3$ & 11.37 \\
$C_{k}$ & constant for determination of eddy diffusivities [1] & $0.1-0.8$ & 3.87 \\
$\tau_{s t}$ & optical thickness of stratus clouds [1] & $5-15$ & 3.47 \\
$\Gamma_{0}$ & parameter of the temperature height dependence (lapse rate) $\left[10^{-3} \mathrm{~K} / \mathrm{m}\right]$ & $4.8-7.2$ & 3.13 \\
\hline
\end{tabular}

Table 2. Set of the anthropogenically influenced parameters $P_{A 5}$ of the monsoon model that are chosen for the deterministic parameter screening. In the last column, an upward pointing arrow $(\uparrow)$ denotes an increasing anthropogenic effect on the parameters and a downward pointing arrow $(\downarrow)$ a decreasing effect.

\begin{tabular}{lllll}
\hline & parameter [unit] & range [unit] & ref. value & \\
\hline$\tau_{s t}$ & optical thickness of stratus clouds [1] & $5-15$ & 7.5 & $\uparrow$ \\
$T_{o c}$ & temperature at the ocean boundaries [K] & $298-303$ & 300 & $\uparrow$ \\
$p C \mathrm{O}_{2}$ & atm. $\mathrm{CO}_{2}$ concentration [ppm] & $300-440$ & 360 & $\uparrow$ \\
$z_{0}$ & surface roughness length for vegetated soil [m] & $0.01-0.8$ & 0.1 & $\downarrow$ \\
$b_{c s}$ & parameter of the clear-sky albedo [1] & $0.02-0.07$ & 0.05 & $\uparrow$ \\
\hline
\end{tabular}

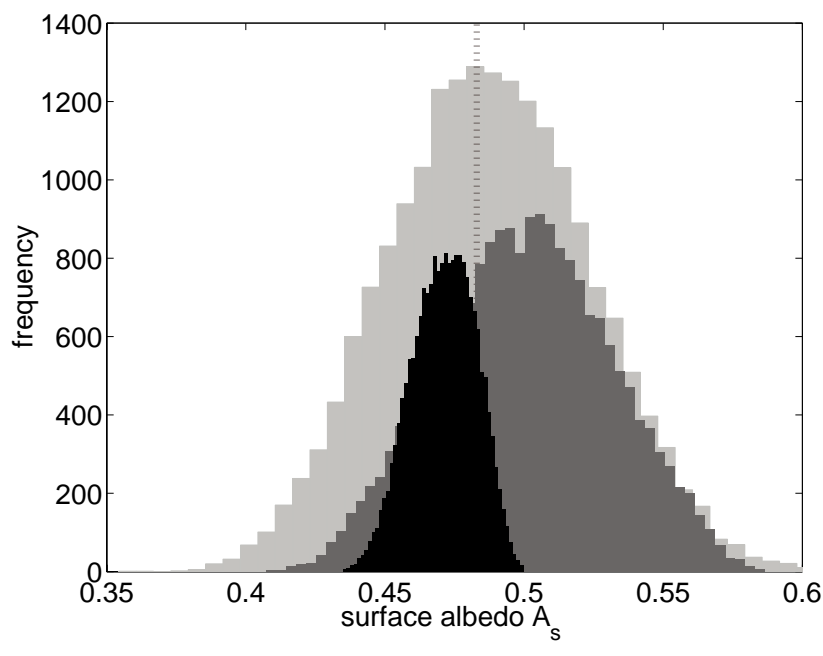

Fig. 6. Results from the Monte Carlo analyses for the whole parameter set $P_{38}$ (light grey), for the five most influential parameters $P_{S 5}$ (dark grey), and the five anthropogenically influenced parameters $P_{A 5}$ (black) all sampled with a Latin hypercube scheme. The figure displays the frequency of the value of the surface albedo $A_{S}$ at the bifurcation point. For $P_{38}$ the mean value is $A_{s}=0.487$, for $P_{S 5}: A_{s}=0.50$, and for $P_{A 5}: A_{s}=0.474$. The vertical dotted line at $A_{S}=0.483$ marks the default value of $A_{S}$, cf. Fig. 2 .

Fig. 6). The standard deviation is $\sigma=0.04$ which corresponds to about $10 \%$ of the mean value. The $5 \%$ and $95 \%$ quantile are at $A_{s}=0.56$ and $A_{s}=0.43$. For the subset $P_{A 5}$ the distribution is shifted a bit to lower values for $A_{s}$ and shows a much smaller standard deviation of $\sigma=0.012$ around a mean value of $A_{s}=0.474$. The parameter set $P_{S 5}$, however, covers a large range and particularly the part of the higher values for the albedo of the total uncertainty range of $P_{38}$.

It is sensible to discuss these results in relation to today's value for the surface albedo over India and to possible changes in this value. The present-day value of the surface albedo derived from satellite data (MODIS, 2006) is approximately 0.164 for summer conditions. The surface albedo has a possible range from $A_{s}=0.12$ for evergreen broadleaf trees to $A_{s}=0.3$ for desert (Dickinson et al., 1986). Hence, even conversion of the total land-cover to desert would not lead to a transgression of the bifurcation point.

\subsection{Deterministic parameter screening}

We perform the deterministic parameter screening (cf. Fig. 3c) as an in-depth parameter uncertainty analysis to investigate how the model behaviour changes in dependence of certain parameters. From the GSA we have deduced the qualitative ranking of the parameters with respect to their influence on the location of the saddle node bifurcation. To make sure that the most important parameters are depicted but that the sample space is not too large to hamper the interpretation of results, the set of the five most influential parameters $P_{S 5}$ is chosen for the analysis (cf. Table 1). 


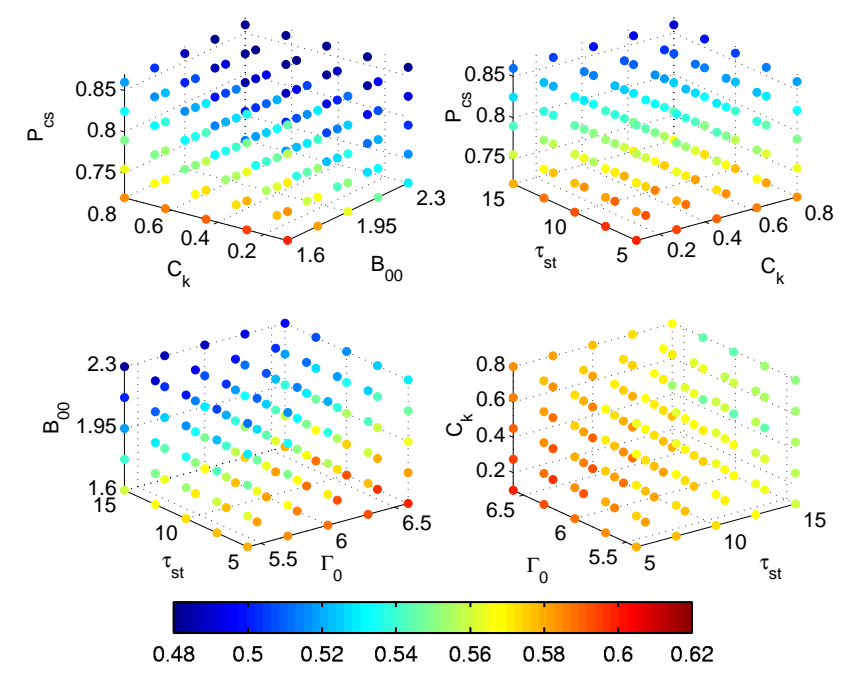

Fig. 7. Maximum value for the surface albedo $A_{S}$ at the saddle node bifurcation in dependence of the parameter set $P_{S 5}$. The colour represents the maximum value of the surface albedo $A_{s}$ at the bifurcation point over those two dimensions that are not shown. The units for the parameters are as in Table 1.

We separate each parameter range from $P_{S 5}$ into $m=4$ equidistant intervals. The resulting $5^{5}$ grid represents a sample with 3125 sample points where all of the corresponding model runs show the saddle-node bifurcation. To visualise this 5-D parameter space we hold constant three parameters in each case. We calculate the maximum and minimum, respectively over the 25 values - corresponding to the other two dimensions that are not shown - for the bifurcation parameter $A_{s}$ at each point in the 3-D space. In total we obtain $103-\mathrm{D}$ plots for the maximum and the minimum, respectively. We have chosen four in each case to illustrate the results (Figs. 7 and 8). From this analysis we can conclude, whether the change of the position of the saddle node bifurcation in dependence on one parameter $p_{i}$ is linear (or nonlinear) or whether an increase of $p_{i}$ leads to an increase or decrease of the value for the surface albedo at the bifurcation point.

The direction from a low to a high value for $A_{s}$ in each 3D subspace is the same for the maximum and the minimum. For all 10 parameter combinations the variation in the values is continuous, there is no abrupt change or reversion of the direction of increasing values for $A_{s}$. The range for the surface albedo $A_{s}$ in each 3-D subspace resembles the ranking from Table 1: the higher the rank of the involved parameters the broader is the range for the surface albedo $A_{s}$. Moreover, the cumulated Figs. 7 and 8 show that these five parameters alone cover a large range and particularly the part of the higher values for the albedo of the total uncertainty range evaluated by the MCA (cf. Fig. 6).

The directions of increasing values for $A_{s}$ can be interpreted by analysing the involved processes that are repre-
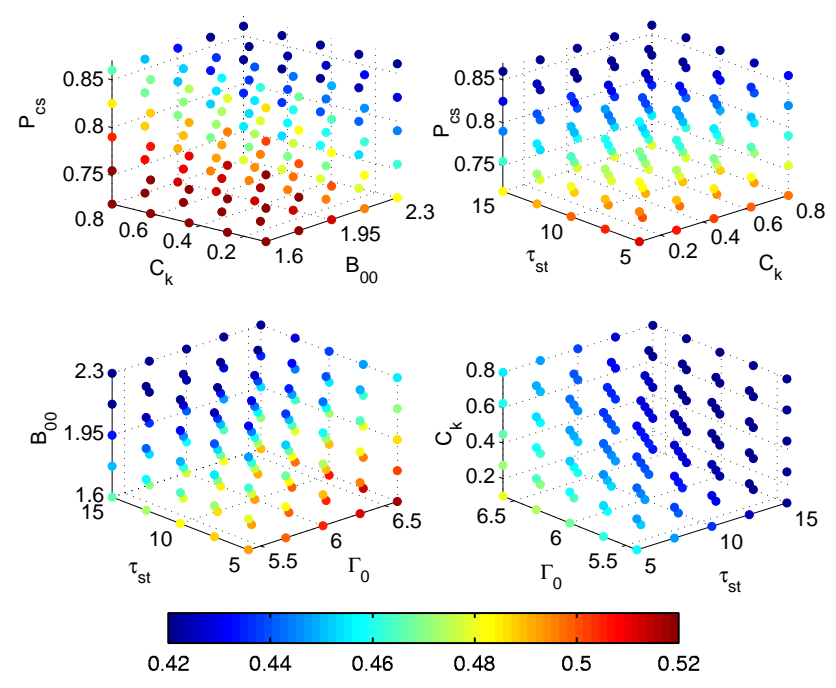

Fig. 8. Same as Fig. 7 but for the minimum of the saddle-node bifurcation.

sented by the parameters. The mechanism for the bifurcation strongly depends on the energy balance over India. All processes affecting this mechanism (e.g. incoming and outgoing solar radiation, advection of heat and moisture) will certainly alter the position of the bifurcation point. The effect of the five most influential parameters can be related to this mechanism: The smaller the albedo of the clear sky (determined by $P_{c s}$, cf. Eq. (8)) and accordingly the planetary albedo, and hence the higher the incoming solar radiation $F_{\downarrow}\left(1-A_{s y s}\right)$, the higher is the amount of available energy. Therefore a higher value for $A_{s}$ is needed to get below the critical value where the monsoon circulation cannot be sustained any more. The heat balance equation (cf. Eq. (1)) explains the influence of the parameter $B_{00}$ that directly influences the outgoing radiation $F_{\uparrow}$ (cf. Eqs. (5) and (6)): the lower $B_{00}$ and hence the outgoing radiation $F_{\uparrow}$, the higher is the available amount of heat and therefore the value for $A_{s}$ at the bifurcation. $C_{k}$ determines the strength of the monsoon circulation and as it enters with the inverse, a higher value for $C_{k}$ results in a lower value for the maximum and minimum at the bifurcation point. The optical thickness of the stratus clouds $\tau_{s t}$ has a similar effect on the albedo of the clouds as $P_{c s}$ on the clear sky albedo, so the stabilising effect leads here as well to higher values for the bifurcation point. The lapse rate coefficient $\Gamma_{0}$ determines the lapse rate $\Gamma$ and therefore the vertical temperature profile (see model description): the higher $\Gamma_{0}$, the lower is the temperature at a specific height $z$ and the lower is the advection of heat by the upper tropospheric circulation from land to the ocean. This implies that more energy is available what shifts the bifurcation point further to higher values of $A_{s}$.

The same analysis can be taken out for the parameter set $P_{A 5}$ listed in Table 2. This investigation will account for the uncertainty in future projections that could alter the monsoon 

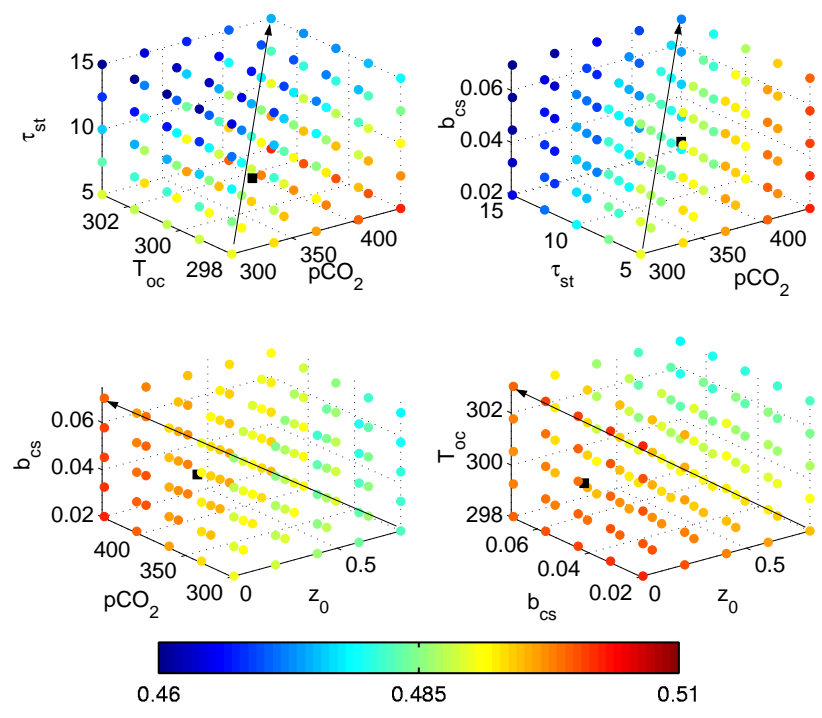

Fig. 9. Same as Fig. 7 but for the parameter set $P_{A 5}$. The black square denotes the present day state, the arrows indicate the direction of a business as usual path of anthropogenic perturbations. The units for the parameters are as in Table 2.

characteristics from a wet to a dry regime. Anthropogenic activities can alter the $\mathrm{CO}_{2}$ concentration by the emission of greenhouse gases. This has an effect on the global mean temperature and therefore on the temperature at the ocean boundaries $T_{o c}$. The ongoing emission of sulphate aerosols has an increasing effect on the albedo of the clear sky $b_{c s}$ and on the optical thickness of the stratus clouds $\tau_{s t}$. Land use or land cover change can alter the surface roughness $z_{0}$ of India, for instance by decreasing the roughness due to forest conversion.

From Figs. 9 and 10 it can be seen that the anthropogenic influence on the two parameters $z_{0}$ and $p \mathrm{CO}_{2}$ shifts the bifurcation point further away from today's values. On the other hand, the increasing values of $\tau_{s t}, b_{c s}$, and $T_{o c}$ due to human activity lead to a decrease of the value for $A_{s}$ at the bifurcation point. The destabilising effect of increasing the albedo parameters $\tau_{s t}$ and $b_{c s}$, which determine the albedos of the clouds and the clear sky $A_{s t}$ and $A_{c s}$ (cf. Eq. 8), leads to a lower amount of available energy and therefore lower value for $A_{s}$ at the bifurcation point, as discussed before in connection with the parameter $P_{c s}$. The influence of the ocean temperature $T_{o c}$ is not continuously increasing or decreasing but shows a turning point at $T_{o c}=300 \mathrm{~K}$ that is due to some symmetry effect of the temperature $T_{a}$ around $T_{o c}=300 \mathrm{~K}$. The stabilising effect of increasing $\mathrm{CO}_{2}$ is related to the decrease of the outgoing radiation $F_{\uparrow}$, that is negatively correlated with $\mathrm{CO}_{2}$ concentration (cf. Eqs. 5 and 6), so that more energy is available resulting in a shift of the bifurcation to higher values.

From Figs. 9 and 10 it can be deduced that it strongly depends on the exact value the five parameters will take in the
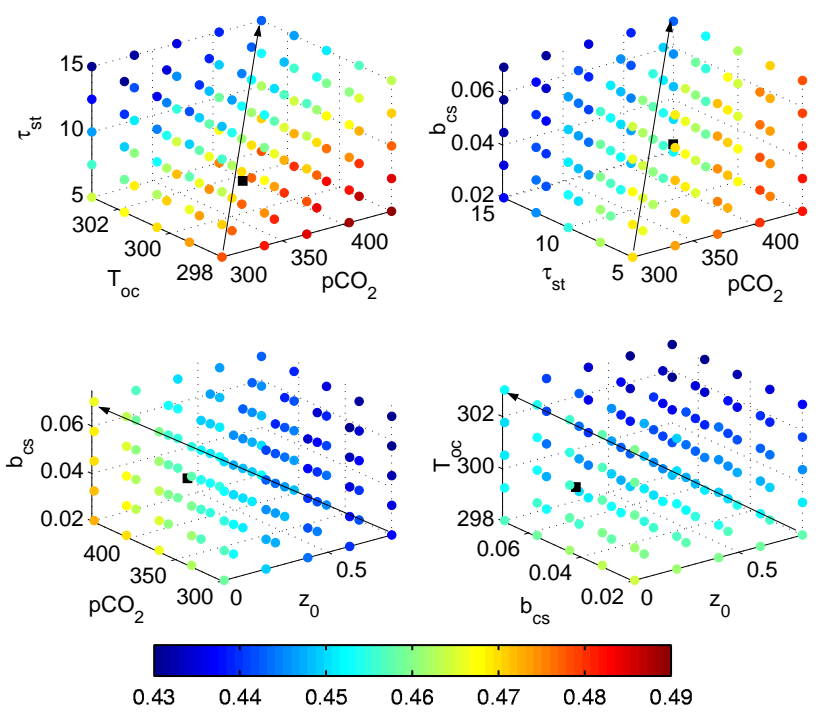

Fig. 10. Same as Fig. 9 but for the minimum values.

future to decide whether anthropogenic action will shift the bifurcation point nearer to today's values.

\section{Conclusions}

A new method was developed and applied for investigating the robustness of a bifurcation point against parameter uncertainties in a model of the Indian summer monsoon. This method builds on combining a bifurcation analysis with a common sensitivity and uncertainty analysis in the high dimensional parameter space. Such an in-depth parameter uncertainty analysis of a bifurcation point is a novel approach and a universal method that can be applied to many other models of similar complexity, where the robustness of a bifurcation point against parameter uncertainties is of interest. The multi-run environment SimEnv with its simple model interface and post-processing techniques has proven to be a valuable tool for this kind of study.

As an example for an application of this method we have analysed a reduced form model of the Indian summer monsoon that shows a saddle node bifurcation in dependence of the surface albedo and other parameters that influence the heat budget of the system. As the bifurcation goes along with a crucial transition from a wet to a dry monsoon regime, we were interested in the estimation of the robustness of the bifurcation point under parameter uncertainty. The methods that were performed for global sensitivity and uncertainty studies are based on a large number of model runs and a tool for post-processing the results. The multi-run environment SimEnv with its simple model interface and post-processing techniques has shown to be highly suitable for this kind of study. 
The multi-parameter uncertainty analysis has impressively shown that the occurrence of the saddle node bifurcation in this model is a very robust feature. In over $96 \%$ of all runs for the Monte Carlo analysis and in 93\% of all runs for the global sensitivity analysis the model shows the abrupt transition from a wet to a dry monsoon regime. The propagation of the parameters uncertainties, however, leads to a very broad range for the surface albedo $A_{s}$ at the bifurcation point indicating a high uncertainty about the actual position of the bifurcation point in phase space.

The global sensitivity analysis shows that the investigated parameters differ largely concerning their influence on the position of the bifurcation point. The most influential parameters are related to the planetary albedo, the incoming and outgoing solar radiation, and the lapse rate. We have also analysed the influence of anthropogenically affected parameters that have opposite effects on the position of the bifurcation point relative to present-day. Consequently, a robust conclusion concerning the influence of human activity cannot be given here but requires model experiments with plausible scenarios of future changes.

The fact that the bifurcation is robust against parameter uncertainties supports earlier findings in Zickfeld et al. (2005). The interpretation in terms of whether the regime transition can be reached under global change is twofold: on the one hand - under the assumption that the model is reliable - the uncertainty analysis shows that the bifurcation point is far away from today's values and that we can be very certain that we will not reach this point in the near future. On the other hand - under the assumption that the model is just a conceptual one that lacks some mechanisms and will not be able to give an estimate of the true value of $A_{s}$ at the bifurcation point - this analysis shows that we have to deal with a wide uncertainty range. Under these uncertainties it is hardly possible to decide how far away we are from the bifurcation. The proximity of the system to such a threshold could e.g. be estimated by means of an analysis that builds on a shift of the spectrum of observational data when coming closer to the bifurcation point (Held and Kleinen, 2004).

These results suggest the need for further investigations with a more complex model, such as a high resolution GCM, to come up with more robust conclusions about the actual point of this possible transition. Furthermore, as we have only investigated the sensitivity of the bifurcation against parameter uncertainties, a next step will be a structural uncertainty analysis where different parameterisations and mechanisms are analysed.

Acknowledgements. Part of this work was funded by the German Research Association (DFG) (PR1175/1-1). B. K. acknowledges support from the Rosa Luxemburg Foundation.

Edited by: U. Feudel

Reviewed by: two referees

\section{References}

Budyko, M.: The Earth's climate, vol. 29 of International Geophysics Series, Academic Press, New York, 1982.

Burns, S., Fleitmann, D., Matter, A., Kramers, J., and Al-Subbary, A.: Indian Ocean climate and an absolute chronology over Dansgaard/Oeschger events 9 to 13, Science, 301, 1365-1367, 2003.

Campolongo, F., Cariboni, J., Saltelli, A., and Schoutens, W.: Enhancing the Morris Method, in Sensitivity Analysis of Model Output. Proceedings of the 4th International Conference on Sensitivity Analysis of Model Output (SAMO 2004), pp. 369-379, Los Alamos National Laboratory, Los Alamos, 2005.

Dickinson, R. E., Henderson-Sellers, A., Kennedy, P., and Wilson, M.: Biosphere-Atmosphere Transfer Scheme (BATS) for the NCAR Community Climate Model, Tech. rep., NCAR/TN275+STR, NCAR, Boulder, Colorado, 1986.

Doedel, E. J.: AUTO: A program for the automatic bifurcation analysis of autonomous systems, in: Proceedings of the 10th Manitoba Conference on Numerical Mathematics and Computing, pp. 265-284, University of Manitoba, Winnipeg, Canada, 1981.

Flechsig, M., Böhm, U., Nocke, T., and Rachimow, C.: Techniques for Quality Assurance of Models in a Multi-Run Simulation Environment, in: Sensitivity Analysis of Model Output, Proceedings of the 4th International Conference on Sensitivity Analysis of Model Output (SAMO 2004), edited by K. M. Hanson and F. M. Hemez, pp. 297-230, Los Alamos National Laboratory, Los Alamos, USA, 2005.

Forest, C., Stone, P., Sokolov, A., Allen, M., and Webster, M.: Quantifying Uncertainties in Climate System Properties with the Use of Recent Climate Observations, Science, 295, 113-117, 2002.

Gross, T. and Feudel, U.: Generalized models as a universal approach to the analysis of nonlinear dynamical systems, Physical Review E, 73, 016 205, 2006.

Gupta, A., Anderson, D., and Overpeck, J.: Abrupt changes in the Asian southwest monsoon during the Holocene and their links to the North Atlantic Ocean, Nature, 421, 354-357, 2003.

Hansen, J., Russell, G., Rind, D., Stone, P., Lacis, A., Lebedeff, S., Ruedy, R., and Travis, L.: Efficient three-dimensional global models for climate studies: Models I and II, Mon. Wea. Rev., 11, 609-662, 1986.

Held, H. and Kleinen, T.: Detection of climate system bifurcations by degenerate fingerprinting, Geophys. Res. Lett., 31, L23 207, doi:10.1029/2004GL020972, 2004.

Kalnay, E., Kanamitsu, M., Kistler, R., Collins, W., Deaven, D., Gandin, L., Iredell, M., Saha, S., White, G., Woollen, J., Zhu, Y., Chelliah, M., Ebisuzaki, W., Higgins, W., Janowiak, J., Mo, K. C., Ropelewski, C., Wang, J., Leetmaa, A., Reynolds, R., Jenne, R., and Joseph, D.: The NMC/NCAR 40-Year Reanalysis Project, Bull. Amer. Meteor. Soc., 77, 437-471, data available at http://www.cdc.noaa.gov/, 1996.

Knutti, R., Stocker, T. F., Joos, F., and Plattner, G.-K.: Constraints on radiative forcing and future climate change from observations and climate model ensembles, Nature, 416, 719-723, 2002.

Kripalani, R. H., Kulkarni, A., Sabade, S. S., and Khandekar, M. L.: Indian Monsoon Variability in a Global Warming Scenario, Natural Hazards, 2003.

Kumar Mittal, A., Dwivedi, S., and Chandra Pandey, A.: Bifurcation analysis of a paradigmatic model of monsoon prediction, Nonlin. Processes Geophys., 12, 707-715, 2005, 
http://www.nonlin-processes-geophys.net/12/707/2005/.

Kuznetsov, Y. A., Levitin, V. V., and Skovoroda, A.: Continuation of stationary solutions to evolution problems in CONTENT, Tech. Rep. AM-R9611, Centrum voor Wiskunde en Informatica, Amsterdam, The Netherlands, 1997.

Longworth, H., Marotzke, J., and Stocker, T. F.: Ocean Gyres and Abrupt Change in the Thermohaline Circulation: A Conceptual Analysis, J. Clim., 18, 2403-2416, 2005.

May, W.: Simulated changes of the Indian summer monsoon under enhanced greenhouse gas conditions in a global time-slice experiment, Geophys. Res. Lett., 29, 1118-1121, 2002.

McKay, M. D., Beckman, R., and Conover, W. J.: A comparison of three methods of selecting values of input variables in the analysis of output from a computer code, Technometrics, 21, 239-245, 1979.

Meehl, G. A. and Washington, W. M.: South Asian Monsoon variability in a model with doubled atmospheric carbon dioxide concentration, Science, 260, 1101-1104, 1993.

MODIS:http://modis-atmos.gsfc.nasa.gov/ALBEDO/index.html, 2006.

Morris, M. D.: Factorial sampling plans for preliminary computational experiments, Technometrics, 33, 161-174, 1991.

Patra, P. K., Behera, S. K., Herman, J. R., Maksyutov, S., Akimoto, H., and Yamagata, T.: The Indian summer monsoon rainfall: interplay of coupled dynamics, radiation and cloud microphysics, Atmos. Chem. Phys., 5, 2181-2188, 2005,

http://www.atmos-chem-phys.net/5/2181/2005/.
Saltelli, A., Tarantola, S., Campolongo, F., and Ratto, M.: Sensitivity Analysis in Practice. A Guide to Assessing Scientific Models, John Wiley \& Sons, 2004.

Schneider von Deimling, T., Held, H., Ganopolski, A., and Rahmstorf, S.: Climate sensitivity estimated from ensemble simulations of glacial climate, Clim. Dyn., 27, 149-163, 2006.

SimEnv: http://www.pik-potsdam.de/software/simenv, 2006.

Stainforth, D. A., Aina, T., Christensen, C., Collins, M., Faull, N., Frame, D. J., Kettleborough, J. A., Knight, S., Martin, A., Murphy, J. M., Piani, C., Sexton, D., Smith, L. A., Spicer, R. A., Thorpe, A. J., and Allen, M. R.: Uncertainty in predictions of the climate response to rising levels of greenhouse gases, Nature, 433, 403-406, 2005.

Zickfeld, K.: Modelling large-scale singular climate events for Integrated Assessment, Ph.D. thesis, Universität Potsdam, Germany, http://deposit.ddb.de/cgi-bin/dokserv?idn=971983453, 2004.

Zickfeld, K., Knopf, B., Petoukhov, V., and Schellnhuber, H.-J.: Is the Indian summer monsoon stable against global change?, Geophys. Res. Lett., 32, L15 707, doi:10.1029/2005GL022771, 2005.

Zwiers, F. W. and Kharin, V. V.: Changes in the extremes of the climate simulated by CCC GCM2 under $\mathrm{CO}_{2}$ doubling, J. Clim., 11, 2200-2222, 1998. 